\title{
Percepção de pacientes hipertensos sobre a doença e motivação para mudança de hábitos
}

\author{
Mônica de Fátima Freires da Silva \\ Gina Andrade Abdala \\ Elisabete Agrela de Andrade \\ Alexsandro dos Santos Machado \\ Maria Dyrce Dias Meira
}

\section{RESUMO}

O objetivo deste estudo foi conhecer as percepções de pacientes hipertensos sobre a doença e a influência de uma intervenção educativa na adoção de hábitos saudáveis relacionados aos "Oito Remédios Naturais". Estudo desenvolvido em abordagem qualitativa por meio de entrevistas semiestruturadas com oito participantes que vivenciarem uma intervenção educativa que aplicou oficinas sobre hábitos saudáveis relacionados aos "Oito Remédios Naturais". Para análise utilizou-se a técnica do Discurso do Sujeito Coletivo, fundamentada na Teoria da Representação Social, que permitiu a representação de 15 Ideias Centrais, entre as quais se destacam: "Antes das oficinas a qualidade de vida era ruim"; "Conscientização dos riscos da hipertensão e motivação para adotar hábitos saudáveis"; "Percepção de benefícios físicos, emocionais e espirituais após as oficinas"; "Ingestão de mais água, devido ao incentivo nas oficinas"; "Dificuldade quanto à temperança e o sono, pela complexidade em romper velhos hábitos"; "A motivação foi aprender sobre os remédios naturais" e "A confiança em Deus aumenta a motivação para se cuidar". Os participantes reconheceram os riscos da hipertensão, demonstrando compreender a influência da adoção de hábitos saudáveis no controle e prevenção de suas complicações. Reconheceram também a complexidade dos determinantes sociais implicados neste contexto.

Palavras-chave: Hipertensão; Promoção da saúde; Educação em saúde; Estilo de vida saudável.

\section{ABSTRACT}

\section{Perception of hypertensive patients about the disease and motivation to chan- ge habits}

The objective of this study was to know the perceptions of hypertensive patients about the disease and the influence of an educational intervention in the adoption of healthy habits related to the "Eight Natural Remedies." The study developed was a qualitative approach through semi-structured interviews with eight participants who experienced an educational intervention that applied workshops on healthy habits related to the "Eight Natural Remedies." For analysis, the Collective Subject Discourse technique was used, based on the Social Representation Theory, which allowed the representation of 15 Central Ideas, among which the following stand out: "Before the workshops, the quality of life was poor"; "Awareness of the risks of hypertension and motivation to adopt healthy habits"; "Perception of physical, emotional and spiritual benefits after the workshops"; "Ingestion of more water, due to the incentive in the workshops"; "Difficulty intemperance and sleep, due to the complexity of breaking old habits"; "The motivation was to learn about natural remedies" and "Trust in God increases the motivation to take care of yourself." The participants recognized the risks of hypertension, demonstrating an understanding that adopting healthy habits influences in the control and prevention of its complications. They also recognized the complexity of the social determinants involved in this context.

Keywords: Hypertension; Health Promotion; Health Education; Healthy Lifestyle.

\author{
Sobre os autores \\ M. F. F. S \\ http://orcid.org/0000-0002 \\ 9400-9924 \\ Centro Universitário Adventista \\ de São Paulo - UNASP - São \\ Paulo, SP \\ mofreires@gmail.com
}

\section{G. A. A.}

http://orcid.org/0000-0001-

8015-0743.

Centro Universitário Adventista de São Paulo - UNASP - São

Paulo

ginabdala@gmail.com

\section{A. S. M.}

http://orcid org/0000-0002-

5335- 5417.

Centro Universitário Adventista de São Paulo - UNASP - São

Paulo, SP

elisabeteagrela1@gmail.com

\section{D. D. M}

http://orcid.org/0000-0003-

1889-9707.

Universidade Federal Rural de Pernambuco - UFRPE - Recife, PE

alexdesapucaia@gmail.com

http://orcid.org/0000-00016313-4637.

Centro Universitário Adventista de São Paulo - UNASP - São

Paulo, SP

dyrcem@yahoo.com.br

\section{Direitos Autorais}

Este é um artigo de acesso aberto e pode ser reproduzido livremente, distribuído, transmitido ou modificado, por qualquer pessoa desde que usado sem fins comerciais. $O$ trabalho é disponibilizado sob a licença Creative Commons CC-BY-NC.

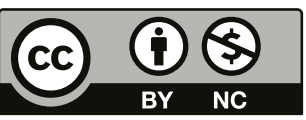




\section{H* INTERACÃO EM PSICOLOGIA}

As Doenças Crônicas Não Transmissíveis (DCNT) foram apontadas como associadas às causas de mortes de $41 \mathrm{mi}-$ Ihões de pessoas em 2016. Destacaram-se as doenças cardiovasculares como responsáveis por 17,9 milhões dessas mortes, em uma faixa etária considerada, cada vez mais, prematura e produtiva, pois tem atingido pessoas a partir dos 30 anos de idade (World Health Organization, 2018). Entre as doenças cardiovasculares, a Hipertensão Arterial Sistêmica (HAS) é a mais prevalente. Segundo dados do Inquérito Nacional de Saúde nas capitais brasileiras e no Distrito Federal a prevalência da autorreferida foi de $24,1 \%$, sendo significativamente associada ao estilo de vida (Malta et al., 2017).

A melhoria das condições de vida individual e coletiva para reduzir os riscos à saúde constitui um grande desafio e impõe o planejamento de ações intersetoriais, que devem ser construídas em parceria com todos os envolvidos na produção do cuidado em saúde (Ministério da Saúde, 2018). Essa construção depende das condições de vida das pessoas e todos os significados implicados neste contexto diante dos fatores que influenciam no processo saúde-doença, segundo o perfil epidemiológico da população brasileira (Malta et al., 2018).

0 plano de ações estratégicas para o enfrentamento das DCNT no Brasil propõe, no eixo promoção de saúde e entre seus objetivos, "|Estudo descritivo e exploratório, desenvolvido em abordagem qualitativa, fundamentado na Teoria da Representação Social (TRS) (Moscovici, 2000/2015) no qual foram realizadas entrevistas semiestruturadas e analisadas com base na técnica do Discurso do Sujeito Coletivo (DSC) (Lefèvre, 2017).

Cabe destacar que o presente estudo integra uma pesquisa maior, com desdobramento em duas etapas. Sendo que, na etapa pertinente a esta fase, utilizou-se como estratégia, as Oficinas de Espiritualidade e Saúde (OES) propostas por Abdala et al., (2015) para orientar a adoção de hábitos saudáveis relacionados aos ORN. Na primeira reunião com o grupo selecionado, foi apresentado a proposta das oficinas. Após terem aceitado o convite e assinado o Termo de Consentimento Livre e Esclarecido (TCLE), considerando as recomendações de pesquisas que envolvem seres humanos, foi pactuado a questão do sigilo e respeito às opiniões divergentes.

Na reunião inicial estiveram presentes nove pessoas, sendo que apenas uma não retornou para os próximos encontros realizados em oito semanas consecutivas, nos meses de maio e junho de 2016. Em cada reunião, foram aplicadas atividades educativas, desenvolvidas com o apoio de uma equipe interdisciplinar, na qual os participantes foram orientados e estimulados a praticarem os hábitos saudáveis sem, contudo, interromper a terapia medicamentosa prescrita.

A finalização das OES marcou o início da avaliação subjetiva para apreender, em uma perspectiva qualitativa, a per- cepção dos hipertensos sobre as contribuições da intervenção educativa quanto à adoção de hábitos saudáveis. Trata-se de uma amostra intencional que incluiu pacientes com hipertensão arterial, cadastrados e acompanhados em uma Unidade Básica da Saúde (UBS) da Regional da Estratégia Saúde da Família (ESF), localizada na Zona Sul do Município de São Paulo.

Foram realizados os contatos telefônicos com os pacientes hipertensos para agendar as entrevistas. No momento do contato, foi estabelecido uma interação entre o pesquisador e o participante, propiciada por uma conexão pré-entrevista conforme recomendação de Minayo (2014). As entrevistas foram agendadas em um único dia, em horários diferentes e aplicadas individualmente após concordância dos participantes de que seus depoimentos seriam gravados para posterior transcrição e análise.

A coleta de dados foi realizada por meio de um questionário contendo as variáveis sociodemográficas e uma entrevista com roteiro semiestruturado contendo quatro questões norteadoras: 1- Como você se sentia sendo uma pessoa com pressão alta, antes das Oficinas de Espiritualidade e Saúde? E agora, o que mudou?; 2- Quais remédios naturais você adotou com maior facilidade? Por quê?; 3- Quais remédios naturais você teve maior dificuldade em adotar? Por quê?; e 4- 0 que mais motivou você a participar das oficinas?

O conteúdo das entrevistas foi armazenado e organizado no software DSCsoft (Instituto de Pesquisa do Discurso do Sujeito Coletivo \& Tolteca, 2018) que facilitou a aplicação da técnica de análise do Discurso do Sujeito Coletivo (Lefèvre, 2017). No tratamento dos dados identificou-se as Expressões-Chave (EC) e agrupou-as conforme as Ideias Centrais (IC), sendo então separadas em categorias. É importante ressaltar que essa técnica de análise permite incluir a representação do mesmo participante em mais de uma categoria, pois ele pode apresentar mais de uma opinião a respeito daquilo que está sendo comentado. Resumindo, a partir do agrupamento das EC, se obtém o DSC e ao mesmo tempo a representação coletiva dos indivíduos da pesquisa (Lefèvre \& Lefèvre, 2014; Lefèvre, 2017).

A técnica do DSC possibilita a apresentação de uma síntese, elaborada com partes de diversos depoimentos reunidos em um só discurso. 0 discurso é construído na primeira pessoa do singular como se fosse a fala de apenas um indivíduo. É uma técnica que se alia à TRS em uma abordagem psicossocial (Lefèvre \& Lefèvre, 2014; Lefèvre, 2017) e busca apreender as crenças e valores que influenciam os comportamentos e que também são influenciados por eles. A TRS amplia a compreensão do significado, atribuído pelos participantes da pesquisa, ao avaliar subjetivamente a realidade vivenciada em sua concretude (Minayo, 2014; Moscovici, 2000/2015). 


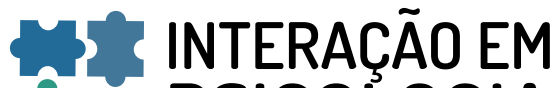 PSICOLOGIA}

Este estudo atendeu todas as normas éticas de pesquisa, envolvendo seres humanos conforme resolução 466/2012 e foi aprovado pelo Comitê de Ética em Pesquisa da Instituição preponente sob parecer $n^{\circ} 688.878$, em 05.06.2014 (CAAE $n^{\circ}$ 20774113.8.0000.5377) e da Secretaria Municipal da Saúde de São Paulo sob o n 818.071, em 05.11.2014 (CAAE $n^{\circ}$ 28905914.3.0000.5377).

\section{RESULTADOS E DISCUSSÃO}

Participaram deste estudo oito pacientes com hipertensão arterial, sendo que sete eram do sexo feminino, seis com idade entre 65 e 80 anos. Em relação a cor da pele referida, seis declararam-se brancas. Quanto à escolaridade, seis deles haviam cursado o primeiro grau completo. Outra variável estudada foi a religião, com predominância de quatro participantes católicos, seguidos de três evangélicos e um espírita.

A análise do conteúdo discursivo dos participantes permitiu as representações sociais sobre a hipertensão e estilo de vida saudável das quais se converteram em quatro categorias. No diagrama abaixo, encontram-se as categorias e as respectivas Ideias Centrais (IC) (Figura 1).

As percepções dos participantes sobre o significado quanto as implicações da hipertensão na maneira como viviam antes e após as OES, apresentadas na Tabela 1, demonstraram a importância do aprendizado sobre os fatores associados que direta ou indiretamente interferem nos desfechos da doença.

Figura 1. Representações Sociais: Categorias e Ideias Centrais

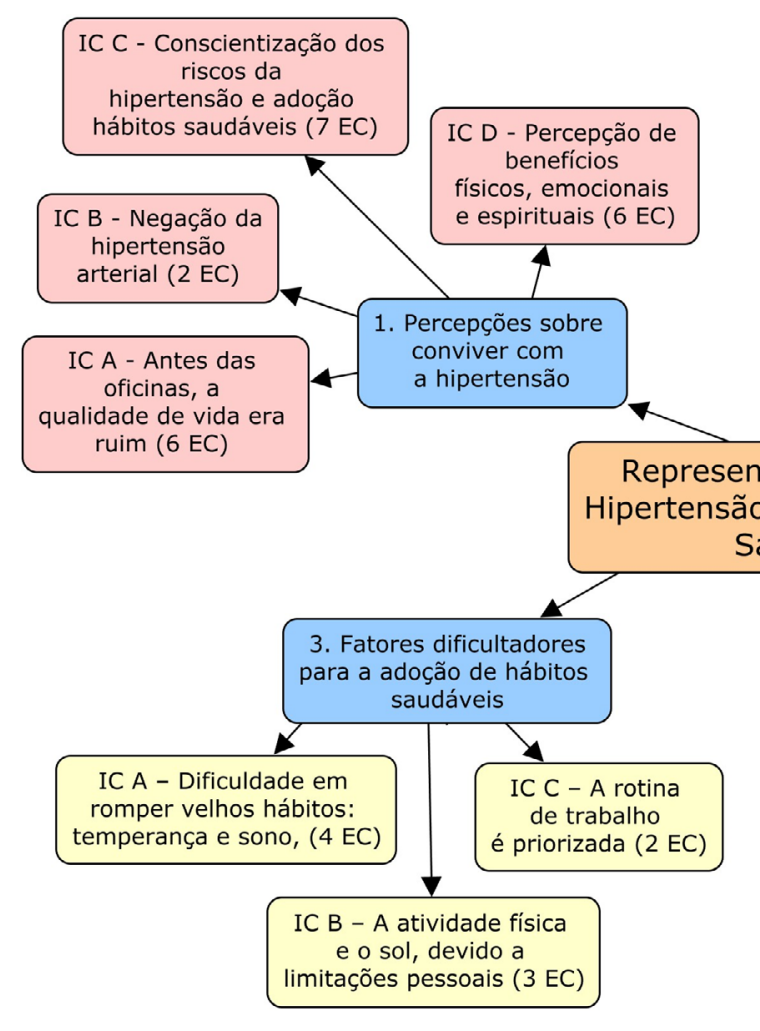

O discurso apresentado na IC A traz representações sobre costumes e crenças sobre alimentos estimulantes, comportamento sedentário, relação com a cultura local, desinformação sobre a doença e depressão. Todos são fatores de risco que interferem no tratamento da HAS e estão relacionados às principais causas modificáveis da doença (Arnett et al., 2019). Os níveis elevados de ansiedade e estresse físico, mental e psicológico contribuem para a elevação da pressão arterial e podem ser melhorados por meio de tratamento não medicamentoso, baseado na mudança do estilo de vida. No caso de depressão, o foco do tratamento é a promoção do bem-estar e autoconfiança (Malachias et al., 2016).

No discurso referente à IC B foram identificados três momentos que antecedem a aceitação de um tratamento: a negação da doença, expressada pela fala "Eu nem tenho pressão 
Tabela 1. Percepções sobre conviver com a hipertensão

\begin{tabular}{|c|c|}
\hline Ideia Central - IC & Discurso do Sujeito Coletivo - DSC \\
\hline IC A - 6 EC & DSC \\
\hline $\begin{array}{l}\text { Antes das oficinas a qua- } \\
\text { lidade de vida era ruim. }\end{array}$ & $\begin{array}{l}\text { Antes de participar grupo eu estava com astral baixo. Eu ficava meio angustiada, eu } \\
\text { era muito tensa, sem esperança, sem vontade das coisas. A pressão estava sempre } \\
\text { alta. Eu não tinha uma orientação para alimentação, para comer pouco sal e gordu- } \\
\text { ra, eu não sabia... Pra mim só a medicação era suficiente. Eu comia muita besteira, } \\
\text { muito doce... Tinha dia que eu não tomava nem um copo de água. Eu tomava o copo } \\
\text { cheio de café e muito chá mate que tem cafeína. Não sabia da importância da ativi- } \\
\text { dade física e não tinha o hábito de fazer exercício. }\end{array}$ \\
\hline IC B - 2 EC & DSC \\
\hline $\begin{array}{l}\text { Negação da hipertensão } \\
\text { arterial. }\end{array}$ & $\begin{array}{l}\text { Olha, na realidade minha pressão não é alta, isso eu nunca tive, né? Mas tenho histó- } \\
\text { rico na família. Na realidade eu tenho, porque eu tomo "lozartana" e se eu fico sem } \\
\text { remédio ela sobe. É que eu não queria aceitar, mas minha família toda tem diabetes } \\
\text { e pressão alta... }\end{array}$ \\
\hline IC C - 7 EC & DSC \\
\hline $\begin{array}{l}\text { Conscientização dos } \\
\text { riscos da hipertensão e } \\
\text { adoção hábitos saudá- } \\
\text { veis. }\end{array}$ & $\begin{array}{l}\text { Esse grupo me incentivou... eu aprendi muitas coisas boas. Depois das oficinas mu- } \\
\text { dou bastante, percebi a diferença. Abriu mais a minha mente, deu essa clareza de } \\
\text { que para a gente viver bem a gente tem que se alimentar, fazer exercício físico, ter } \\
\text { disciplina. Tenho mais consciência do que é uma pressão alta e do que ela causa, né? } \\
\text { Aprendi que a pessoa tem que descansar um pouco, então eu melhorei o tempo de } \\
\text { sono e foi muito benéfico! Eu diminuí o café e a comilança durante o dia. Tô comen- } \\
\text { do mais legumes, até salada. Eu já parei com aquele excesso de doces e tô tirando } \\
\text { fritura... Agora, também, eu tomo mais água e procuro fazer exercícios. Tô pegando } \\
\text { mais Sol, porque eu quase não pegava, né? A Confiança em Deus eu acho que sem- } \\
\text { pre tive... Então, eu senti que mudanças simples de hábitos são difíceis, são bem } \\
\text { difíceis! Mas eu tô mudando aos poucos, e agora, com fé em Deus eu vou melhorar. }\end{array}$ \\
\hline IC D - 6 EC & DSC \\
\hline $\begin{array}{l}\text { Percepção de benefícios } \\
\text { físicos, emocionais e es- } \\
\text { pirituais. }\end{array}$ & $\begin{array}{l}\text { Depois que comecei aqui (oficina) eu mudei o comportamento porque às vezes a } \\
\text { gente tem aquela agitação, né? Aprendi algumas técnicas de relaxamento, simples e } \\
\text { que qualquer um pode executar e é de grande benefício. Senti melhora na disposição } \\
\text { e estou mais calma, mais tranquila... Senti redução das dores no corpo e estou dor- } \\
\text { mindo melhor. Percebi que a minha aparência tá mais alegre, tenho mais ânimo, mais } \\
\text { vontade de fazer as coisas. Sinto paz espiritual, sabe? Eu percebi a diferença. A últi- } \\
\text { ma vez minha pressão estava } 12 \times 7 \text {, e eu diminui o peso. Participar das oficinas me } \\
\text { deu um ganho. A gente conhece novas pessoas, a gente não conversava no posto, } \\
\text { agora a gente se encontra e conversa sempre, mudou tudo isso. Minha família notou } \\
\text { a diferença, notou que a gente não fica mais só, né? }\end{array}$ \\
\hline
\end{tabular}

alta"; negação com uma certa abertura para tomada de consciência "mas na realidade eu tenho, porque eu tomo "lozartana", em seguida toma consciência e aceita a hipertensão "É que eu não queria aceitar". Todo esse processo sucede no pensamento. A HAS é considerada uma doença silenciosa e agressiva e, normalmente, ocorre a negação como forma de defesa na tentativa de evitar o confronto com a realidade, sendo que a força que a ameaça exerce pode variar de uma pessoa para outra, tanto quanto a resposta que cada indivíduo oferece a essa força ou ameaça (Lefèvre \& Lefèvre, 2015).

Percebe-se no discurso apresentado na IC C, que os participantes reconhecem o valor da interação que se estabeleceu no grupo, deixando claro que o apoio social, promovido durante os encontros, favoreceu o aprendizado e, consequentemente, motivou a adoção de hábitos saudáveis. Evidências apontam 


\section{M." INTERACÃO EM PSICOLOGIA}

que mulheres apoiadas socialmente apresentam disposição para saírem de casa e realizarem atividade física em grupo. Elas ampliam a rede de amizades e melhoram os aspectos físicos, emocionais e sociais (Agostinho et al., 2020).

Destaca-se, no DSC (IC C), uma representação que se ancora na expressão "Então... Eu senti que mudanças simples de hábitos são difíceis, são bem difíceis!". Nesse sentido, é importante destacar que entre os valores e princípios que embasam as ações da Política Nacional de Promoção da Saúde (PNPS) estão o "reconhecimento da singularidade das pessoas e o processo de atenção e cuidado em defesa da saúde e da vida". As ações de promoção da saúde devem levar em consideração também a autonomia sem, contudo, ignorar a complexidade de fatores socioambientais, econômicos, políticos e culturais que influenciam suas escolhas e modo de viver (Ministério da Saúde, 2018). Nesse DSC percebe-se o reconhecimento da complexidade envolvida no desejo de mudar os hábitos nocivos à saúde que, por sua vez, estão interligados aos fatores que coexistem no âmbito individual e social e são determinantes no processo saúde-doença (Pettres \& Da Ros, 2018).

Os participantes fazem referência à confiança em Deus como sendo uma força aglutinadora no processo de mudança de hábitos. Sobre essa força, investigações científicas indicam que pessoas com fé em um "Ser Superior" apresentam maior equilíbrio para lidar com o estresse da vida cotidiana; melhor recuperação de depressão; menos emoções negativas e meIhor socialização ou adaptação (Koenig, 2007). Os estudos sobre a influência da espiritualidade na saúde têm avançado e, nesta direção, destaca-se uma revisão integrativa que incluiu 30 publicações, realizadas entre os anos de 2011 e 2016. Os resultados apontaram a relevância da dimensão espiritual nos cuidados com a saúde e ressaltaram a fé como único apoio para o enfrentamento de algumas doenças, incluindo as cardiovasculares e suas consequências para a vida diária. Os autores apontaram, ainda, a ação influenciadora da confiança em Deus para minimizar os agravos dos agentes estressores que permeiam as relações sociais e familiares (Thiengo et al., 2019).

O papel do coping espiritual, em pessoas idosas hospitalizadas, foi reafirmado na prática do cuidado por Esperandio et al. (2019) ao abordarem a importância de se utilizar estratégias que valorizam essa dimensão. Nesse momento da vida, que é considerado de grande vulnerabilidade, a espiritualidade assume um importante papel nos desfechos da saúde. Os participantes perceberam benefícios físicos, emocionais e espirituais após as oficinas e relataram as diferenças promovidas pela prática dos hábitos saudáveis aprendidos.

No discurso correspondente à IC D, os participantes fazem referência aos valores da pressão arterial após participarem das oficinas. Os dados apresentados por Santos (2018), que avaliou os resultados da intervenção educativa sobre os marcadores biológicos e a pressão arterial nos mesmos participantes do presente estudo, demonstraram uma redução, em média, de $2 \mathrm{mmHg}$, tanto na pressão sistólica como na diastólica. A diferença na pressão diastólica final do grupo que participou da intervenção foi considerada estatisticamente significante $(p=0,038)$ em relação ao grupo controle. Além da queda nos níveis pressóricos, os participantes afirmaram melhora na qualidade de vida após a intervenção.

Resultados semelhantes foram registrados na pesquisa conduzida por Alves et al. (2016), na qual utilizaram a mesma estratégia de oficinas e registraram uma redução média dos níveis da pressão sistólica de $146 \mathrm{mmHg}$ para $128 \mathrm{mmHg}$ e manutenção da diastólica na média de $80 \mathrm{mmHg}$. Nessa direção, vale destacar uma pesquisa na qual os participantes se submeteram ao programa NEWSTART, desenvolvido em 14 dias em regime de imersão, que possibilitou uma mudança progressiva dos hábitos relacionados aos ORN. Os dados coletados antes e após a intervenção, que envolveu 114 pacientes com hipertensão, resultaram em uma diminuição significativa $(p<0,001)$ da pressão arterial. Os autores destacaram que os medicamentos foram descontinuados ou diminuídos em $93 \%$ dos participantes (Sanchez et al., 2019).

0 aprendizado significativo foi mencionado como motivo para a adoção de hábitos saudáveis e valorizado o incentivo do grupo na tomada de consciência sobre o autocuidado. A interação entre as pessoas suscita manifestações cognitivas e emocionais que resultam em um processo reflexivo, possibilitado pela troca de experiências. Os participantes destacaram ainda, que os resultados positivos foram notados por seus familiares. Barreto e Marcon (2014), enfatizam que a família possui papel fundamental de influenciar quanto à adesão ao tratamento não farmacológico por meio do estímulo ao autocuidado. Por outro lado, podem surgir situações estressantes, inerentes à dinâmica familiar, que podem ser geradoras do desequilíbrio da pressão arterial.

A percepção dos benefícios psicológicos, representados pelo bem-estar físico, emocional e espiritual referidos, evidenciou que o processo educativo, possibilitado pelas oficinas, foi capaz de envolver os participantes, favorecendo as mudanças comportamentais. A PNPS, revisada em 2018, reconhece a importância de se mobilizar todos os atores sociais para sua efetivação, bem como utilizar estratégias de educação em saúde mais abrangentes (Ministério da Saúde, 2018).

A Tabela 2 traz a representação dos participantes sobre os aspectos que contribuíram para promover a adoção de alguns hábitos saudáveis. 
Tabela 2: Fatores facilitadores para adoção de hábitos saudáveis

\begin{tabular}{|c|c|}
\hline Ideia Central - IC & Discurso do Sujeito Coletivo - DSC \\
\hline$I C A-6 E C$ & $D S C$ \\
\hline $\begin{array}{l}\text { Ingestão de mais água, } \\
\text { devido ao incentivo nas } \\
\text { oficinas. }\end{array}$ & $\begin{array}{l}\text { DSC - Acho que principalmente a água foi a mais fácil. Sempre ouvi falar da água, mas } \\
\text { nunca levei a sério, eu tinha o hábito de tomar pouca. Depois dos slides que o menino } \\
\text { mostrou foi joia. Hoje em dia eu tomo bastante água, porque está me fazendo muito } \\
\text { bem. Eu levanto cedinho e tomo um copo aí vou para caminhada, levo a garrafinha para } \\
\text { completar os } 8 \text { copinhos do dia. Agora eu tô com o hábito, e também estou dando água } \\
\text { pro meu pessoal lá de casa. }\end{array}$ \\
\hline$I C B-4 E C$ & $D S C$ \\
\hline $\begin{array}{l}\text { Conscientização quanto } \\
\text { à exposição ao sol, ao ar } \\
\text { puro e a atividade física. }\end{array}$ & $\begin{array}{l}\text { Eu tinha mais dificuldade antes, agora não, depois da conscientização da importância } \\
\text { da exposição solar, agora estou tomando o sol pela manhã, ou eu tiro uma parte do } \\
\text { horário do almoço para exposição à Luz solar, pelo menos quinze minutos. Respiração } \\
\text { profunda, o ar puro e a caminhada, eu faço tudo junto. Eu consegui incluir porque vou } \\
\text { para o parque todos os dias. }\end{array}$ \\
\hline$I C C-3 E C$ & $D S C$ \\
\hline $\begin{array}{l}\text { Alimentação saudável, } \\
\text { por sentir os benefícios. }\end{array}$ & $\begin{array}{l}\text { A alimentação eu tive mais consciência. Agora compro mais fruta. Tô sentindo diferen- } \\
\text { ça, porque com o estômago cheio eu tinha muitos pesadelos. Também tô fazendo chá } \\
\text { de ervas caseiras. O único que não tirei lá de casa foi o doce, o açúcar para adoçar as } \\
\text { coisas, mas agora eu uso pouco. Quando meu filho adoça as coisas, eu falo: "olha que } \\
\text { a diabete está aí dentro!". Então, eu estou influenciando minha família e minha vizinha } \\
\text { também. }\end{array}$ \\
\hline$I C D-3 E C$ & $D S C$ \\
\hline $\begin{array}{l}\text { A confiança em Deus, por } \\
\text { sentir gratidão. }\end{array}$ & $\begin{array}{l}\text { A confiança em Deus eu sempre tive, esse remédio é o maior de todos, está em pri- } \\
\text { meiro lugar, né? E, depois de ver as meninas participando com a gente eu fiquei mais } \\
\text { apegada. Eu tenho muita fé em Deus. Esse já vive na mente, porque se não fosse Deus } \\
\text { eu não existia, acho que eu não estava nem aqui mais, viu? Agradeço todos os dias e } \\
\text { digo: "obrigado, Senhor". }\end{array}$ \\
\hline$I C E-1 E C$ & $D S C$ \\
\hline $\begin{array}{l}\text { O sono, por compreender } \\
\text { a importância. }\end{array}$ & $\begin{array}{l}\text { Dos oito elementos, o que eu adotei com mais facilidade foi a higiene do sono. Esse } \\
\text { horário eu via como lazer, mas eu estava estragando o momento de descanso, pois eu } \\
\text { começava a ficar mais ligado e agora eu tô ficando mais relaxado... Tomei consciência } \\
\text { quando foram expostos os elementos e a importância do sono. }\end{array}$ \\
\hline
\end{tabular}

O discurso relacionado à IC A traz representações sobre incentivo e estímulos que perpassa por um processo de autoconhecimento abrindo um acesso facilitador para o hábito diário, até que este seja incorporado à rotina. Por exemplo, a água, foi incorporada à rotina dos participantes e de acordo com o estudo de Rondon et al. (2020), essa prática resulta na redução do sobrepeso e obesidade. Outro estudo relata que um tratamento hidroterápico controla os níveis pressóricos em pessoas hipertensas (Silva et al. 2020).

Os benefícios demonstraram que as práticas adotadas induziram melhora da qualidade de vida, possibilidade de maior independência e autonomia e, também, sentimento de prazer em realizar as atividades propostas (Silva et al., 2016a).
No discurso da IC B a representação que se mostra é a conscientização a partir das orientações que tiveram durante a intervenção educativa. Embora as condições de vida para mudança de hábito sejam complexas, podem ser estimuladas empregando estratégias de educação e saúde. Os participantes deste estudo, puderam vivenciar estímulos diferentes para alcançarem uma consciência crítica para adesão das práticas de saudáveis. Além disso, ampliaram seus conhecimentos sobre os benefícios das mudanças do estilo de vida, com ênfase na promoção de saúde (Macedo et al., 2020).

$O$ discurso apresentado na IC C traz evidências de representações ligadas à consciência dos benefícios da alimentação saudável. 0 pensamento coletivo se caracteriza em poli- 
fasia cognitiva, imagem mental das diferentes modalidades do pensamento e conhecimento. Essas modalidades são extraídas das culturas específicas, das experiências sociais, suas intenções, suas expectativas e sua compreensão da situação, além disso, formam padrões de interdependência exercendo influência mútua uns sobre os outros e juntos geram novos padrões de conhecimento, crenças e imagens (Marková, 2017). Ressalta-se que a memória de momentos significativos é uma função cognitiva fundamental no processo ensino e aprendizagem, bem como para a definição da identidade de uma pessoa. É na memória que se registra as vivências, por meio dela acontece a interação e socialização entre as pessoas pela possibilidade de transmitir experiências (Casemiro et al., 2018).

No discurso apresentado na IC D a espiritualidade emergiu, provavelmente, devido a escuta qualificada que fica evidente na expressão "E, depois de ver as meninas [as pesquisadoras] participando com a gente eu fiquei mais apegada". Este recurso é apresentado por Maynart et al. (2014) como sendo uma técnica de estabelecimento de vínculos respeitosos, de acolhimento, de cuidado integral com foco na integralidade de uma pessoa.
Por meio desta ferramenta o paciente se sente ouvido e apoiado. Isso o faz gostar de quem o escuta, podendo ser decisivo no sucesso do tratamento. Hefti e Esperandio (2016, p. 14), com uma visão holística do paciente, discorrem em seu estudo sobre as questões do "cuidado espiritual como componente importante a ser integrado na atenção ao paciente".

$\mathrm{O}$ discurso correspondente à IC E tem como representação o aprendizado e a compreensão dos benefícios do sono, conforme referencial apresentado a seguir. Corrêa et al. (2018) descrevem a importância da conscientização da higiene do sono sobre o risco de desenvolver e ou agravar doenças cardiovasculares, acidentes de trabalho, desempenho acadêmico entre outros agravantes. $O$ estudo mostra que é alta a prevalência dos distúrbios do sono nos EUA e que no Brasil pelo menos $76 \%$ da população são acometidas por, pelo menos, um sintoma.

$\mathrm{Na}$ Tabela 3 os participantes expressam de maneira contundente as implicações dos determinantes sociais e como eles acarretam limitações pessoais para adoção de novos hábitos que interferem em suas rotinas de vida e trabalho.

Tabela 3: Fatores dificultadores para adoção de hábitos saudáveis

\begin{tabular}{l|l}
\hline Ideia Central - IC & Discurso do Sujeito Coletivo - DSC \\
\hline IC A - 4 EC & DSC \\
\hline
\end{tabular}

É difícil a temperança. O mais difícil foi deixar de tomar café. Comecei alguns dias com chazinho, mas aí... não consegui deixar o cafezinho. Café é vício, eu acho... é algo do meu dia a dia. Então, o sono também foi difícil porque o meu

Dificuldade em romper vesono fica descontrolado. E, quando eu penso muito, o sono some.

Ihos hábitos: temperança e sono.

Eu quero deixar de fazer algumas coisas, então eu digo que não vou fazer e de repente eu vou e faço! Por exemplo: a abstinência do cigarro, porque é assim, você vai me entender: eu só fumo quando estou em crise. Eu fiquei praticamente um ano inteiro depressiva, então eu fumei um tempo maior e ficou um pouco mais difícil, eu não parei totalmente.

\begin{tabular}{l|l}
\hline IC B - 3 EC & DSC \\
\hline & Pra mim a atividade física está sendo o mais difícil, ainda não dá pra fazer, meu
\end{tabular}

A atividade física e o sol, devido às limitações pessoais. marido depende de mim pra tudo. As caminhadas ainda não consegui fazer, até me movimento, mas tenho dores nas pernas. Eu também não estou tomando sol, porque fica ardendo um pouco. É que eu não gostava de jeito nenhum do sol, porque eu peguei muito sol lá no Norte e fiquei receosa do sol daqui também.

DSC

\begin{tabular}{l|l}
\hline IC C - 2 EC & DS \\
\hline
\end{tabular}

A rotina de trabalho é priorizada.

A alimentação foi difícil também por conta da minha profissão. Então eu passo muito da hora do almoço. Às vezes eu não tomo nem água. Tem dia de comer só meia noite. $\mathrm{E}$ aí o médico me falava: leva uma fruta e fala com a cliente que vai ali... e come alguma coisa. Mas, ainda não consegui pôr em prática. 


\section{INTERACÃO EM LF PSICOLOGIA}

Mônica de Fátima Freires da Silva, Gina Andrade Abdala, Elisabete Agrela de Andrade, Alexsandro dos Santos Machado e Maria Dyrce Dias Meira
Os participantes reconheceram a complexidade de se romper com hábitos arraigados, praticados durante toda sua vida. A concepção da promoção da saúde a partir da determinação social rompe com a prática que reduz o pensar crítico e a dominância cultural para uma visão ampliada de saúde de forma integral, dialogada e transformadora, baseada nas implicações dos determinantes sociais do processo saúde-doença, que se faz dependente da educação em saúde pautada na realidade de vida de cada indivíduo (Pettres \& Da Ros, 2018).

No discurso referente à IC A, apresentado na Tabela 3 , as representações giram em torno de crenças cristalizadas a favor de velhos hábitos como demonstram as referências a seguir. Uma das diretrizes apresentada pela Sociedade Brasileira de Cardiologia (SBC), para a prevenção e controle da HAS, propõe incentivar esses pacientes a aderirem ao tratamento não medicamentoso baseado nas práticas de um EVS, como coadjuvante ao tratamento medicamentoso. Embora os autores reconheçam que envolvem práticas, aparentemente, simples elas esbarram em forte resistência por exigir mudanças comportamentais que, normalmente, estão relacionadas à hábitos arraigados (Malachias et al., 2016).

Dias et al. (2015) consideram que uma abordagem bem estruturada para o "tratamento não medicamentoso" poderia incentivar os hipertensos a reduzirem a ingestão excessiva de álcool e sal, abandonar o sedentarismo e incluir em seu cardápio o consumo de vegetais e consequentemente controlar o peso. Consideram ainda que as mudanças comportamentais são fundamentais e que intervenção nessa direção são de baixo custo, não oferecem riscos e são eficazes como medidas para redução da pressão arterial.
No DSC apresentado na IC B percebe-se que a representação envolve os fatores pessoais e emocionais como limitantes à adoção de novos hábitos para a saúde, os quais apontam o estresse emocional como um dos fatores do desencadeamento e manutenção da hipertensão. Consideram que ele atua também como barreira para a adesão ao tratamento e às mudanças de hábitos, principalmente no caso de idosos que demandam intervenções mais adequadas (Pinto et al., 2020).

A representação exposta na IC C, do DSC correspondente, desvela o quanto as influências da vida contemporânea que envolve a pessoa em prioridades materiais e pode ser um impeditivo para adesão a um EVS ao deixar a saúde em segundo plano. Por se tratar de uma doença multifatorial, a responsabilidade do controle da HAS não pode ser atribuída apenas ao paciente. A adesão ao tratamento depende de fatores que vão além da própria doença, envolvendo os serviços de saúde, profissionais, sociedade, família e condições de trabalho e de vida (Barreto \& Marcon, 2014; Lopes et al., 2015; Mantovani et al., 2016). Um estudo qualitativo que entrevistou feirantes com hipertensão apresentou que, na percepção deles, se trata de uma doença crônica, silenciosa, traiçoeira, que mata; que viver atarefado e estressado afeta a pressão e que pode levar a morte. Além disso, manifestaram o entendimento que o controle da HAS exige mudanças de hábitos (Silva et al., 2015).

$\mathrm{Na}$ Tabela 4 são apresentadas as percepções dos participantes quanto às contribuições das Oficinas de Espiritualidade e Saúde na mobilização de recursos que podem impactar positivamente na adoção dos hábitos saudáveis. 
Tabela 4. Contribuições das Oficinas de Espiritualidade e Saúde

\begin{tabular}{|c|c|}
\hline Ideia Central - IC & Discurso do Sujeito Coletivo - DSC \\
\hline IC A - 3 EC & DSC \\
\hline $\begin{array}{l}\text { O acolhimento e o apoio } \\
\text { social mobilizam a moti- } \\
\text { vação. }\end{array}$ & $\begin{array}{l}\text { Foi muito bom ter aceitado participar da pesquisa, não tinha nada a perder. Vi que } \\
\text { seria uma coisa legal. E foi muito bom. Eu acho que o grupo foi a maior força, o } \\
\text { benefício maior foi o companheirismo. Foi muito bom mesmo! Me fizeram esque- } \\
\text { cer as preocupações. As amizades, as meninas todas foram muito legais mesmo. } \\
\text { Exatamente isso, foi o apoio social mesmo! Eu achei fantástico! }\end{array}$ \\
\hline IC B - 5 EC & DSC \\
\hline $\begin{array}{l}\text { A motivação foi aprender } \\
\text { sobre os remédios natu- } \\
\text { rais. }\end{array}$ & $\begin{array}{l}\text { Participar das oficinas foi uma benção na minha vida, eu amei! E aprendi muito! } \\
\text { Gostei das palestras, das explicações de tudo, né? Foi fantástico! Os remédios } \\
\text { naturais que eles passaram pra gente, são tudo coisa fácil que a gente tem em } \\
\text { casa, e a gente não usa. Olha... Vou ser sincera... O que foi falado a gente até já } \\
\text { sabe, só que é muito bom alguém bater na tecla, falando... principalmente por } \\
\text { profissionais, pois a gente só vê remédio, a droga... E, o remédio natural a gente } \\
\text { não vê. São coisas fáceis, basta querer praticar. Quando a gente tem consciência } \\
\text { aí a gente dá valor. Valeu a pena! E eu até faria de novo para aprender ainda mais. }\end{array}$ \\
\hline IC C - 5 EC & DSC \\
\hline $\begin{array}{l}\text { A confiança em Deus au- } \\
\text { menta a motivação para } \\
\text { se cuidar. }\end{array}$ & $\begin{array}{l}\text { O que de fato me motivou a continuar nas oficinas foi a confiança em Deus, e } \\
\text { essa vontade imensa que eu tô de mudar de vida porque eu tô com pressão alta. } \\
\text { A confiança de Deus é um dos remédios mais importantes na minha vida! Eu par- } \\
\text { ticipei das oito oficinas. Se estava chovendo eu estava aqui, se estava frio ou com } \\
\text { vento de rachar, eu vinha porque eu queria ficar no grupo pra poder saber mais. É } \\
\text { bom na vida a gente aprender! E esse aprendizado vou levar comigo! }\end{array}$ \\
\hline
\end{tabular}

A IC A correspondente ao DSC apresentado na Tabela 4 apresenta o acolhimento e o apoio social como fator capaz de mobilizar estímulos internos que podem auxiliar a adoção de hábitos saudáveis. Sato e Ayres (2015) ao estudaram o acolhimento diferenciado praticado em uma UBS que, por meio da arte, restaurou o significado do acolhimento humanizado possibilitado pelo apoio grupal. Além disso, resgatou a dignidade dos participantes e enriqueceu recursos subjetivos para a percepção ampliada de novas possibilidades do cuidado à saúde.

No discurso correspondente à IC B, a representação focalizou a satisfação de aprender sobre os RN, como um fator de motivação para participar das oficinas. Os resultados do estudo de Casemiro et al. (2018) apontaram que quando existe um interesse natural pela temática abordada as pessoas se sentem estimuladas a aprender. As motivações mais relevantes, normalmente, são aquelas ligadas a fatores internos, como memória afetiva. São motivações que não exigem recompensa exterior, pois elas são focadas na execução da ação. Outro estudo, nessa direção, demonstra que um dos motivos para o desenvolvimento e a manutenção de ações grupais está representado pela construção de saberes, promovido pela troca de experiências, o aprendizado e o empoderamento quanto ao cuidado com a saúde (Friedrich et al., 2018).

$\mathrm{O}$ discurso referente à IC $\mathrm{C}$ traz a representação de que a confiança em Deus os motivou a participarem das oficinas e aprender sobre o autocuidado. As pessoas, de maneira geral, gostariam que os médicos dessem mais abertura aos pacientes para abordarem sobre os valores, crenças e experiências religiosas e espirituais e, também, que houvesse sensibilidade e respeito independente da orientação religiosa dos médicos (Moreira-Almeida et al., 2016).

Tomkins et al. (2015) destacaram que todas as atividades de fé religiosas estimulam a crença em um Deus, ou em um ser superior, que pode influenciar na prevenção e tratamento da doença. Juntamente com o apoio psicossocial os aspectos espirituais, como, por exemplo, uma prece, quando oferecidos aos pacientes, normalmente, são aceitos de bom grado, ressaltando que em alguns países essas práticas ainda não são aceitas.

A promoção da saúde conforme preconizado por Lefèvre e Lefèvre (2015) propõe uma postura que pode contribuir para 


\section{MLE INTERACÃO EM PSICOLOGIA}

desconstruir a ideia de mundo sem doentes, modelo biomédico tradicional, para uma visão de mundo sem doenças. Ao propor intervenções que vão além da saúde física viabiliza o resgate da consciência crítica; do pensar e agir lógico e agregador; do senso da subordinação equivocada e das contradições da vida moderna impostas. Ao promover o discernimento sobre o que é bom ou ruim, a interrelação pessoal provinda do diálogo para a busca do saber e a constância no fazer, se traduz em uma consciência de mundo, de si mesmo e do outro e restabelece a voz para o enfrentamento dos determinantes sociais e o resgate da dignidade humana.

Com relação às limitações deste estudo, é possível destacar que ele não é causal e houve um número reduzido de pessoas com hipertensão que aceitaram participar das oficinas. Por se tratar de uma doença multifatorial, preconiza-se que sejam realizadas novas pesquisas com desenhos metodológicos longitudinais que possibilitem análises mais abrangentes. Ressalta se, ainda, que uma avaliação sobre a consolidação dos hábitos saudáveis adotados, a longo prazo, poderia identificar os aspectos que impactam efetivamente no controle da HAS.

\section{CONSIDERAÇÕES FINAIS}

A representação dos participantes hipertensos, apresentada nos DSCs, quanto às implicações de conviver com a doença e a motivação para adoção de hábitos promotores da saúde integral, apontou para uma qualidade de vida ruim antes da intervenção e melhoras subsequentes. Na percepção dos participantes, os fatores que mais motivaram a adoção de novos hábitos foram: os benefícios físicos, emocionais e espirituais vivenciados durante as OES; incentivo durante as oficinas quanto à conscientização das vantagens de se eliminar os hábitos nocivos à saúde; importância de utilizar os Oito Remédios Naturais, incluindo a gratidão e a espiritualidade. Ressalta-se, que a representação sobre a confiança em Deus desperta uma linha de raciocínio de que esta é uma dimensão que ajuda a mudar o pensamento e progressivamente os levam à adoção de hábitos mais saudáveis.

Os participantes reconheceram que foram influenciados por uma corrente condutora na qual a espiritualidade foi capaz de incentivá-los a adotarem um estilo de vida mais saudável. A intervenção educativa foi uma estratégia que se mostrou capaz de promover a saúde, pois conquistaram comportamentos positivos e, mesmo esbarrando em fatores complexos que limitam a mudança de hábitos, o processo educativo possibilitou ampliar as escolhas para uma saúde integral

Romper com paradigmas determinantes de velhos hábitos e ter um bom manejo dos fatores pessoais e emocionais, que limitam a adoção de novos hábitos, foram as principais dificuldades apontadas. Reconheceram a complexidade dos determinantes sociais relacionadas às limitações pessoais e condições socioeconômicas, implicadas no contexto de vida, ao afirmarem "Eu senti que mudanças simples de hábitos são difíceis, são bem difíceis! Mas eu tô mudando aos poucos...". A despeito disso, os resultados das falas apresentadas nos DSC mostraram que as vivências grupais promoveram maior equilibrio emocional e motivaram as mudanças comportamentais dos participantes

A associação dos hábitos saudáveis vivenciados com as melhorias da saúde, percebida e referida pelos participantes deste estudo, são indícios de que eles começaram a relacionar os comportamentos saudáveis ao bem-estar que sentiram. Ao favorecer a conexão dos saberes construídos a partir da interrelação entre os componentes do grupo, preconiza-se que a técnica das oficinas possibilitou o aprendizado significativo, configurando um fator motivacional para a adoção dos hábitos saudáveis, relacionados aos Oito Remédios Naturais. Com base nos DSCs construídos depreende-se que agregar ao tratamento uma postura holística, humanizada, respeitosa, cuidadosa, responsável e participativa de todos envolvidos no processo de tratamento, produz melhores resultados.

\section{FINANCIAMENTO}

Não houve financiamento de terceiros.

\section{DECLARAÇÃO DA CONTRIBUIÇÃO DOS AUTORES}

Certificamos que todos os autores participaram suficientemente do trabalho para tornar pública sua responsabilidade pelo conteúdo. A contribuição de cada autor é atribuída como se segue: M.F.F.S e M.D.D.M. contribuíram para a administração do projeto, análise formal dos dados, conceitualização, investigação, metodologia e tabulação dos dados; M.F.F.S. foi responsável pela preparação da redação inicial do artigo (rascunho original); M.D.D.M. responsável pela supervisão e redação final (revisão e edição); G.A.A. foi responsável pela tabulação dos dados, visualização do artigo e validação; E.A.A. e A.S.M. foram responsáveis pela visualização do artigo, revisão do artigo e validação.

\section{AGRADECIMENTOS}

Os autores agradecem à Odete Santelle pelo tratamento dos dados, aplicando a técnica do Discurso do Sujeito Coletivo (DSC) com apoio do software DSCsoft. 


\section{DECLARAÇÃO DE CONFLITOS DE INTERESSE}

Os autores declaram que não há conflitos de interesse no manuscrito submetido.

\section{REFERÊNCIAS}

Abdala, G. A., Meira, M. D. D., \& Teixeira, C. A. (2015). Oficinas de Espiritualidade e Saúde: oito semanas de espiritualidade e saúde para hipertensos ou pré-hipertensos. Casa Publicadora Brasileira.

Agostinho, B. K., Silveira, L. M., Matioli, M. R., Bertazone, T. M. A., Matias, V. D., Bueno Júnior, C. R., Stabile, A. M. (2020). Apoio social, qualidade de vida e a percepção de mulheres praticantes de treinamento físico. Research, Society and Development, 9(1), e03911520. http://dx.doi.org/10.33448/ rsd-v9i1.1520

Alves, S. S., Silva, V. V. V., Silva, I. C., Teixeira, C. A., Abdala, G. A. \& Meira, M. D. D. (2016). Oficinas de espiritualidade e saúde para hipertensos ou pré-hipertensos. Lifestyle Journal, 3(2), 69-88. https://doi.org/10.19141/2237-3756/lifestyle.v3.n2. p70-89

Armstrong, T., Bauman, A., Candeias, V., Lewicka, M., Richards, J., \& Schoeppe, S. (2008). Review of best practice in interventions to promote physical activity in developing countries. WHO. https://www.who.int/dietphysicalactivity/bestpracticePA2008.pdf

Arnett, D. K., Blumenthal, R. S., Albert, M. A., Buroker, A. B., Goldberger, Z. D., Hahn, E. J., Himmelfarb, C. D., Khera, A., Lloyd-Jones, D., McEvoy, J. W., Michos, E. D., Miedema, M. D., Muñoz, D., Smith, S. C., Jr., Virani, S. S., Williams, K. A., Yeboah, J., \& Ziaeian, B. (2019). ACC/AHA guideline on the primary prevention of cardiovascular disease: a report of the American College of Cardiology/American Heart Association Task Force on Clinical Practice Guidelines. Circulation, 140(11), 596-646. https://doi.org/10.1161/ CIR.0000000000000678

Barreto, M. S., \& Marcon, S. S. (2014). Patient perspectives on family participation in the treatment of hypertension. Texto \& Contexto - Enfermagem, 23(1), 38-46. https://doi. org/10.1590/S0104-07072014000100005

Brazil. Ministry of Health of Brazil. Health Surveillance Secretariat Department of Surveillance of Noncommunicable Diseases and Health Promotion. (2018). Report on the 3rd Forum on the Strategic Action Plan to Tackle Noncommunicable Diseases in Brazil. http://bvsms.saude.gov.br/bvs/ publicacoes/report_forum_strategic_action_diseases.pdf

Casemiro, F. G., Quirino, D. M., Diniz, M. A. A., Rodriques, R. A. P., Pavarini, S. L., \& Gratão, A. C. M. (2018). Effects of health education in the elderly with mild cognitive impairment Revista Brasileira de Enfermgem, 71(Suppl. 2), 854-863. https://doi.org/10.1590/0034-7167-2017-0032
Corrêa, C. C., Campos, L. D., \& Weber, S. A. T. (2018). Conscientização sobre hábitos relacionados ao Sono no interior do Estado de São Paulo. Archives of Health Investigation, 7(3), 115-117. https://doi.org/10.21270/archi.v7i3.2810

Dias, E. G., Alves, J. C. S., Santos, V. C. O., Aguiar, D. K. A., Martins, P. R., \& Barbosa, M. C. (2015). Lifestyle and hindering factors in controlling hypertension. Revista de Enfermgem da UFPI, 4(3), 24-29. https://doi.org/10.26694/reufpi. v4i3.3526

Esperandio, M. R. G., Escudero, F. T., Fanini, L., \& Macedo, E. P. N. (2019). Envelhecimento e espiritualidade: O papel do coping espiritual/religioso em pessoas idosas hospitalizadas. Interação em Psicologia, 23(2), 268-280. https://doi. org/10.5380/psi.v23i02.65381

Friedrich, T. L., Petermann, X. B., Miolo, S. B., \& Pivetta, H. M. F. (2018). Motivações para práticas coletivas na Atenção Básica: percepção de usuários e profissionais. InterfaceComunicação, Saúde, Educação, 22(65), 373-385. https:// doi.org/10.1590/1807-57622016.0833

Hefti, R., \& Esperandio, M. R. G. (2016). O modelo interdisciplinar de cuidado espiritual - uma abordagem holística de cuidado ao paciente. Horizonte, 14(41), 13-47. https://doi. org/10.5752/P.2175-5841.2016v14n41p13

Instituto de Pesquisa do Discurso do Sujeito Coletivo \& Tolteca. (2018). DSCsoft (Versão desconhecida) [Programa computacional/Software] http://www.tolteca.com.br/dscSoft.aspx

Koenig, H. G. (2007). Religion, spirituality and psychiatry: a new era in mental health care. Revista de Psiquiatria Clinica, 34(Suppl. 1), 5-7. https://doi.org/10.1590/S010160832007000700002

Lefèvre, F. (2017). Discurso do Sujeito Coletivo: nossos modos de pensar, nosso eu coletivo. Andreoli.

Lefèvre, F., \& Lefèvre, A. M. C. (2014). Discourse of the collective subject: social representations and communication interventions. Texto \& Contexto Enfermagem. 23(2), 502-507. https://doi.org/10.1590/0104-07072014000000014

Lefèvre, F., \& Lefèvre, A. M. C. (2015). Promoção de saúde: a negação da negação. Vieira e Lent.

Lopes, J. H. P., Oliveira, A. M. G., Pereira, A. C., \& Meneghim, M. C. (2015). Adesão do paciente à terapia medicamentosa da hipertensão arterial: Revisão da literatura. Revista de Odontologia da Universidade Cidade de São Paulo, 27(3), 235-243. https://doi.org/10.26843/ro_unicid.v27i3.254

Macedo, L. C., Faveri, F. D. P., Gerotto Junior, L. C., Costa, M. P. R., Zanelli, T. L. P., Rodrigues, A. S..., Milagres, C. S. (2020). Abordagem educativa para promoção de saúde em usuários hipertensos: Uma revisão integrativa da literatura. Brazilian Applied Science Review, 4(4), 2678-2690. https://doi. org/10.34115/basrv4n4-040 
Malachias, M. V. B., Franco, R. J. S., Forjaz, C. L. M., Pierin, A. M. G., Gowdak, M. M. G., Klein, M. R. S. T., \& Matsudo, V. (2016). 7th Brazilian Guideline of Arterial Hypertension: Chapter 6 - Non-pharmacological treatment Arquivos Brasileiros de Cardiologia, 107(3, Suppl. 3), 30-34. https://doi. org/10.5935/abc.20160156

Malta, D. C., Oliveira, T. P., Santos, M. A. S., Andrade, S. S. C. A., Silva, M. M. A. (2016). Avanços do Plano de Ações Estratégicas para o Enfrentamento das Doenças Crônicas não Transmissíveis no Brasil, 2011-2015. Epidemiologia e Serviços de Saúde, 25(2), 373-390. https://doi.org/10.5123/ s1679-49742016000200016

Malta, D. C., Bernal, R. T. I., Andrade, S. S. C. A., Silva, M. M. A., \& Velasquez-Melendez, G. (2017). Prevalence of and factors associated with self-reported high blood pressure in Brazilian adults. Revista de Saúde Pública. 51(Suppl. 1), 1-11. http://dx.doi.org/10.1590/s1518-8787.2017051000006

Malta, D. C., Reis, A. A. C., Jaime, P. C., Morais, O. L., Neto, Silva, M. M. A., \& Akerman, M. (2018). O SUS e a Política Nacional de Promoção da Saúde: perspectiva resultados, avanços e desafios em tempos de crise. Ciência e Saude Coletiva, 23(6), 1799-1809. https://doi.org/10.1590/141381232018236.04782018

Malta, D. C., Andrade, S. S. C. A., Oliveira, T. P., Moura, L., Prado, R. R., \& Souza, M. F. M. (2019). Probabilidade de morte prematura por doenças crônicas não transmissíveis, Brasil e regiões, projeções para 2025. Revista Brasileira de Epidemiologia, 22, e190030. https://doi.org/10.1590/1980549720190030

Mantovani, M. F., Mattei, A. T., Rocha, E. N., Major, C. B., Ulbrich, E. M., \& Barboza, K. C. (2016). Motivos para adesão ao tratamento medicamentoso da hipertensão arterial. Saúde (Santa Maria), 42(2), 59-66. https://doi. org/10.5902/2236583421466

Marková, I. (2017). A fabricação da Teoria de Representações Sociais. Cadernos de Pesquisa, 47(163), 358-375. https:// doi.org/10.1590/198053143760

Maynart, W. H. C., Albuquerque, M. C. S., Brêda, M. Z., \& Jorge, J. (2014). A escuta qualificada e o acolhimento na Atenção Psicossocial. Acta Paulista de Enfermagem, 27(4), 300-304. https://doi.org/10.1590/1982-0194201400051

Minayo, M. C. S. (2014). o desafio do conhecimento: pesquisa qualitativa em saúde. (14a. edição.) Hucitec.

Ministério da Saúde. Secretaria de Vigilância em Saúde. Secretaria de Atenção à Saúde. (2018). Política Nacional de Promoção da Saúde: PNPS: Anexo I da Portaria de Consolidação $n^{\circ} 2$, de 28 de setembro de 2017, que consolida as normas sobre as políticas nacionais de saúde do SUS. http://bvsms.saude.gov.br/bvs/publicacoes/politica_nacional_promocao_saude.pdf
Moreira-Almeida, A., Sharma, A., Rensburg, B. J. V., Verhagen, P. J., \& Cook, C. C. H. (2016). WPA Position statement on spirituality and religion in psychiatry. World Psychiatry, 15(1), 87-88. https://doi.org/10.1002/wps.20304

Moscovici, S. (2015). Representações sociais: investigações em psicologia social.

(11a. Edição). Vozes. (Obra original publicada em 2000).

Pettres, A. A., \& Da Ros, M. A. (2018). A Determinação Social da Saúde e a Promoção da Saúde. Arquivos Catarinenses de Medicina, 47(3), 183-196.

Pinto, T. S. B., Pinto, A. S. B., Torres, A. A., Firmino, B. S., Sousa, A. L. A., Bezerra, S. L. (2020, no prelo). A interferência dos fatores psicológicos na hipertensão arterial. Revista inovale, 1(1). https://doi.org/10.29327/515133.1.1-7

Ramos, C. F. V., Araruna, R. C., Lima, C. M. F., Santana, C. L. A., \& Tanaka, L. H. (2018). Education practices: research-action with nurses of Family Health Strategy. Revista Brasileira de Enfermagem, 71(3), 1144-1151. https://doi. org/10.1590/0034-7167-2017-0284

Rondon, M. C. S., Silva, G. S. H., Abdala, G. A., Silva, N. O. D., Abdala, S. A. (2020). La importancia del agua en la vida de los adolescentes. Lecturas: Educación Física y Deportes, 24(260), 78-94 https://doi.org/10.46642/efd.v24i260.1911

Sanchez, A., Chung, S. C., Mejía, A., Ramirez, F. E., Shavlik, G. W., Bivens, R. L, Brown-Fraser, S., Gallant, R. D. (2019). Multiple lifestyle interventions reverses hypertension. Cogent Medicine, 6 (1) 1636534. https://doi.or$\mathrm{g} / 10.1080 / 2331205 \times .2019 .1636534$

Santos, D. C. (2018). Religiosidade, marcadores biológicos e qualidade de vida relacionada à saúde de hipertensos [Dissertação de mestrado],Centro Universitário Adventista de São Paulo. Repositório Institucional do UNASP. https:// biblioteca.sophia.com.br/9198_RI/index.asp?codigo_sophia $=500464$

Sato, M., \& Ayres, J. R. C. M. (2015). Arte e humanização das práticas de saúde em uma Unidade Básica. Interface- Comunicação, Saúde, Educação, 19(55), 1027-1038. https:// doi.org/10.1590/1807-57622014.0408

Silva, S. R. A., Amorim, R. C., Almeida, A. M. (2015). Percepção de feirantes hipertensos sobre o adoecer crônico. Revista Enfermagem UERJ, 23(6), 761-766. https://doi. org/10.12957/reuerj.2015.8494

Silva, M. R., Santos, N. P. V., Santos, R. A., Cunha, G. R., \& Torres, L. M. (2016a). A percepção do idoso institucionalizado sobre os benefícios das oficinas terapêuticas. Revista Brasileira em Promoção da Saúde, 29(Suppl. 1), 76-84. https:// doi.org/10.5020/18061230.2016.sup.p76 
Silva, R. L. D. T., Barreto, M. S., Arruda, G. O., Marcon, S. S. (2016b). Avaliação da implantação do programa de assistência às pessoas com hipertensão arterial. Revista Brasileira de Enfermagem. 69(1), 79-87. https://doi.org/10.1590/ 0034-7167.2016690111i

Silva, L. W. S., Novaes, I. G., Aderne, J. N. N., Almeida, M. A., Santos, L. C. (2020). Aquatic activities: contributions to treatment of individuals with chronic disease . Research, Society and Development, 9(9), e342996927. https://doi. org/10.33448/rsd-v9i9.6927

Thiengo, P. C. S., Gomes, A. M. T., Mercês, M. C., Couto, P. L. S., França, L. C. M., \& Silva, A. B. (2019). Espiritualidade e religiosidade no cuidado em saúde: revisão integrativa. Cogitare Enfermagem, 24, e58692 https://doi.org/10.5380/ ce.v24i0.58692
Tomkins, A., Duff, J., Fitzgibbon, A., Karam, A., Mills, E. J., Munnings, K., Smith, S., Seshadri, S. R., Steinberg, R. V., \& Yugi, P. (2015). Controversies in faith and health care. The Lancet, 386(10005), 1776-17885. https://doi.org/10.1016/S01406736(15)60252-5

White, E. G. (2017). A Ciência do Bom Viver. [10 ed.]. Casa Publicadora Brasileira. (Obra original publicada em 1905).

World Health Organization. (2013). Global action plan for the prevention and control of noncommunicable diseases 20132020. https://www.who.int/nmh/publications/ncd-action-plan/en/

World Health Organization. (2018). World health statistics 2018: monitoring health for the SDGs, sustainable development goals. https://apps.who.int/iris/handle/10665/272596

Submetido em 18/02/2020

Primeira avaliação 29/09/2020

Aceito em 15/10/2020 